Research Article

\title{
Simultaneous Detection of Actinobacillus pleuropneumoniae and Haemophilus parasuis in Pig by Duplex Droplet Digital PCR
}

\author{
Wen Wen, Lei Shi, Xun Chen \\ Research Institute of Food Safety and Nutrition, Jinan University, Guangzhou 510632, China
}

\section{ARTICLE INFO}

\section{Article History}

Received 05 August 2020

Accepted 12 September 2020

\section{Keywords}

Droplet digital PCR

detection

pig

Actinobacillus pleuropneumoniae Haemophilus parasuis

\begin{abstract}
Actinobacillus pleuropneumoniae (A. pleuropneumoniae) and Haemophilus parasuis (H. parasuis) are important pathogens of swine, which cause great economic losses in the swine industry and bring great safety hazards to food safety. In this study, we established a duplex Droplet Digital Polymerase Chain Reaction (ddPCR) assay for sensitive detection of A. pleuropneumoniae and H. parasuis simultaneously in pork. The limit of detection of plasmid DNA could reach 2 copies $/ \mu \mathrm{L}$ for both $A$. pleuropneumoniae and H. parasuis; which were approximately 50 - and 20 -fold greater sensitivity than Quantitative real-time PCR (qPCR). Both qPCR and ddPCR showed high linearity and positive correlation with standards. The detection results of clinical suspected samples for A. pleuropneumoniae and $H$. parasuis indicated the positive detection rate of ddPCR (10.7\% and $12.5 \%)$ was higher than that of qPCR (7.1\% and 8.9\%). Therefore, the duplex ddPCR assay could be used as an effective quantitative detection to diagnose A. pleuropneumoniae and $H$. parasuis simultaneously, which may lay a foundation for the development of substitute rapid diagnostic technique.
\end{abstract}

\section{GRAPHICAL ABSTRACT}

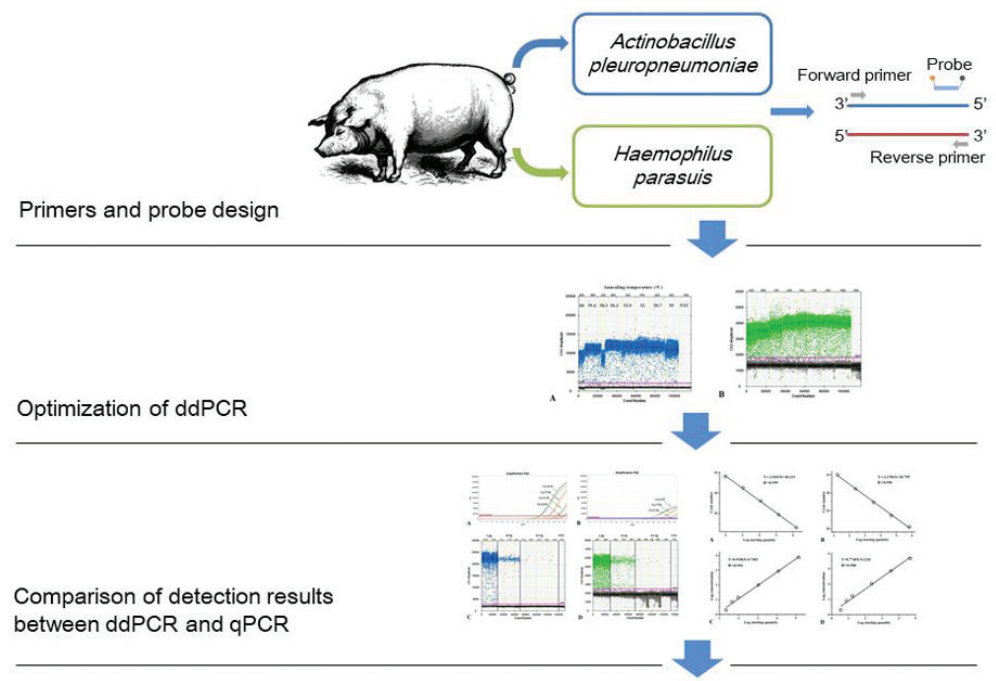

Application and detection of clinical samples

(C) 2020 The Authors. Publishing services by Atlantis Press International B.V. This is an open access article distributed under the CC BY-NC 4.0 license (http://creativecommons.org/licenses/by-nc/4.0/).

\section{INTRODUCTION}

As an important source of protein intake and a national strategic reserve, pork has high nutritional value and is one most important red meat foods in the human diet, and the demand for pork is

"Corresponding author. Email: cx-wolf@163.com

Peer review under responsibility of the International Association of Dietetic Nutrition and Safety increasing globally [1]. In recent years, people pay more attention to food safety, and the safety of meat has always been the focus of societal concerns. The serious meat safety issues may directly lead to the health problems of consumers, and the government needs to recall the contaminated products on the market. When the swine occasionally suffer from certain major diseases, it often changes the demand for pork and further affects the price of pork [2]. Such issues like the outbreak of Streptococcus suis in Sichuan province [3], the swine flu swept the whole world [4], and the African swine 
fever broke out in China [5] happened these years, which caused a serious blow to the swine industry. Then the quality and safety of pork closely related to human health cannot control effectively. It is therefore critical for timely monitoring and prevention of swine epidemics to ensure the safety of pork consumption.

Actinobacillus pleuropneumoniae (A. pleuropheumoniae) is a respiratory pathogen in swine, and it is the pathogenic agent of porcine pleuropneumonia in swine [6]. Haemophilus parasuis (H. parasuis) is the specific pathogenic cause of Glässer's disease in swine, which brings about polyserositis in swine, including pleuritis, peritonitistis and arthritis [7]. Both of them are among the most common bacterial causes of porcine respiratory disease, and often associated with the porcine respiratory disease complex caused by both primary and secondary respiratory pathogens [8]. Although these two pathogens will not affect the food safety directly, the present of A. pleuropheumoniae and H. parasuis in pig can damage the quality and taste of meat, and reduce the edible quality of pork. Furthermore, the porcine respiratory disease is associated with a high incidence rate and fatality rate, which causes substantial losses in the swine industry worldwide and the associated healthcare costs [9-11].

In recent years, Enzyme-linked Immunosorbent Assay (ELISA) [12,13], nested Polymerase Chain Reaction (nested PCR) [14,15] and Quantitative real-time PCR (qPCR) [16-18] have been developed to detect and serotype $H$. parasuis and A. pleuropneumoniae with improved sensitivity and efficiency. However, it is difficult to distinguish the mixed infection of these two diseases by clinical symptoms and pathological features in clinical diagnosis. Thus, multiplex PCR detection has been widely used in many fields in recent years, such as monitoring food pathogenic microorganisms $[19,20]$ and detecting veterinary diagnosis [21-23]. Moreover, Droplet Digital PCR (ddPCR), the latest version of digital PCR, allows better accuracy of DNA quantification [24]. The ddPCR can detect bacterial pathogens with greater sensitivity, which is crucial for early diagnosis and disease control. At present, with the use of commercial ddPCR system such as the Bio-Rad QX100/200, many studies involving duplex quantitative detection have been carried out [25-27]. In this study, we established a duplex ddPCR assay for identifying $A$. pleuropneumoniae and $H$. parasuis simultaneously in comparison with qPCR.

\section{MATERIALS AND METHODS}

\subsection{Strains and Clinical Samples}

Field isolates of A. pleuropneumoniae strains (serotype 1-15), field isolates of $H$. parasuis strains (serotype 1-15), Classical Swine Fever Virus (CSFV), Porcine Circovirus 2 (PCV2), Pseudorabies Virus (PRV), Porcine Reproductive and Respiratory Syndrome Virus (PRRSV), Porcine Epidemic Diarrhea Virus (PEDV), Streptococcus suis (S. suis), Staphylococcus aureus (S. aureus) (ATCC25923), Salmonella Typhimurium (ATCC14028) were obtained from a commercial company (Yongshun Biological Pharmaceutical, Guangdong, China). Fifty lung tissues from 50 healthy piglets in one pig farm and 140 clinical tissue samples (lungs and tonsils) from 10 pig farms were collected from the Institute of Animal Sciences (Guangdong Academy of Agricultural Sciences, Guangdong, China). All clinical samples were stored at $-80^{\circ} \mathrm{C}$ for further use.

\subsection{Nucleic Acid Extraction and Reverse Transcription}

The DNA of A. pleuropneumoniae, H. parasuis, PCV2, PRV, S. suis, S. aureus, Salmonella and the RNA of CSFV, PRRSV and PEDV was extracted by DNA/RNA extraction kit (Magen Bio-tech, Shanghai, China), according to the manufacturer's instruction. And the RNA was reverse transcribed using the PrimerScript RT reagent kit (TaKaRa, Dalian, China). All DNA and cDNA were stored at $-20^{\circ} \mathrm{C}$.

\subsection{Primers and Probes}

The primers and probes of qPCR and ddPCR assays were designed by using Oligo Primer Analysis Software (Molecular Biology Insights, Colorado Springs, CO, USA) then synthesized from a commercial company (Sangon Biotech, Shanghai, China). The probes were labeled with 6-carboxy-fluorescein (FAM) and VIC as the fluorescent reporter, respectively; and minor groove binder (MGB) as the fluorescence quencher. The primers and probe set for A. pleuropneumoniae were designed were as follow: F1 (5'-GCTGACCGCAGTATAACTGTATGG-3'); R1 (5'-CGTCCCCAGTCGTTGATATTAT-3'); and probe (5'-FAM-ATTATTTGGCACTGACGGTG-MGB-3'); for $H$. parasuis were F1 (5'-TCGCCTTGGCCGTAATTCTA-3'); R1 (5'-ACGTAAGCCTTTTCTGTTGTAACATC-3'); and probe (5'-VIC-AAATGATGCAGGATGGG-MGB-3'). The plasmid standards pUC57-APP of A. pleuropneumoniae and pUC57HPS of $H$. parasuis were constructed by inserting the $551 \mathrm{bp}$ sequence of apxIVA (GenBank No. CP030753.1) amplified by primers: FP: 5'-TTGCTGACCGCAGTATAACTGTATG-3'; RP: 5'-GAGGTAAAACAATATATCAATAGCTTAAC-3', and 747 bp sequence of OMP P2 (GenBank No. KU508604.1) amplified by primers: FP: 5'-CTACGGTTTTGGTCGTTATGAG-3'; RP: 5'-GCAGACTATAAATTACATAAACAAGTTGT-3' into commercial vector pUC57 (Sangon Biotech, Shanghai), respectively. And the plasmid pUC57-APP and pUC57-HPS were used as the positive, respectively. The purified recombinant plasmids were quantified (Quibt 3.0; Thermo Fisher Scientific, Waltham, MA, USA), and recalculated to plasmid copies/ $\mu \mathrm{L}$ using an online calculator (available at http://scienceprimer.com/copy-numbercalculator-for-realtime-pcr). The recombinant plasmids were serially 10 -fold and twofold diluted. Then, the dilutions and plasmids were stored at $-20^{\circ} \mathrm{C}$ and $-70^{\circ} \mathrm{C}$, respectively.

\section{4. ddPCR Assay}

A duplex ddPCR assay was designed to amplify A. pleuropneumoniae and $H$. parasuis with primers and probes by using QX200 droplet digital PCR system (Bio-Rad Laboratories, Hercules, CA, USA). In each step of amplification, the optimized $20 \mu \mathrm{L}$ mixtures of duplex ddPCR reaction containing $10 \mu \mathrm{L}$ of $2 \times$ ddPCR supermix for probes (no dUTP; Bio-Rad), $800 \mathrm{nM}$ of each primer, $400 \mathrm{nM}$ of each TaqManMGB probes, and $1 \mu \mathrm{L}$ of each DNA template. $20 \mu \mathrm{L}$ of ddPCR reaction mixtures and $70 \mu \mathrm{L}$ of Droplet Generation oil for Probes (Bio-Rad) were inserted in an eight-well cartridge to generate the droplets. With the use of a droplet generator (Bio-Rad), each sample was divided into 20,000 water-in-oil nanolitre-sized droplets. After that, the $40 \mu \mathrm{L}$ 
generated droplet emulsion was transferred to a new 96-well PCR plate (Eppendorf, Hauppauge, NY, USA), then heat-sealed with a pierceable sealing foil sheet by using the PX1 ${ }^{\text {ma }}$ PCR plate sealer (Bio-Rad) and amplified in C1000 Touch $^{\mathrm{Ts}}$ deep-well thermal cycler (Bio-Rad) finally. The optimized cycling conditions of ddPCR were as follows: $95^{\circ} \mathrm{C}$ for $10 \mathrm{~min}$; then 40 cycles of $94^{\circ} \mathrm{C}$ for $30 \mathrm{~s}, 56.3^{\circ} \mathrm{C}$ for $60 \mathrm{~s}$, and one cycle of $98^{\circ} \mathrm{C}$ for $10 \mathrm{~min}$, and ending at $12^{\circ} \mathrm{C}$.

\section{5. qPCR Assay}

The qPCR assay was performed using ABI QuantStudio 6 Flex realtime PCR system (Thermo Fisher Scientific). The $20 \mu \mathrm{L}$ qPCR reactions comprised $10 \mu \mathrm{L}$ of AceQ qPCR probe master mix (Vazyme, Nanjing, China), $1 \mu \mathrm{L}$ of each DNA template, and the final concentrations of primer and probe were 200 and $100 \mathrm{nM}$, respectively. The reaction conditions of qPCR were as follows: $95^{\circ} \mathrm{C}$ for $5 \mathrm{~min}$, followed by $35 \mathrm{cycles}$ at $95^{\circ} \mathrm{C}$ for $10 \mathrm{~s}$, and $60^{\circ} \mathrm{C}$ for $34 \mathrm{~s}$.

\subsection{Specificity and Reproducibility Test of ddPCR}

The specificity of the ddPCR assay was validated, and nucleic acid extracts of A. pleuropneumoniae, H. parasuis, PCV2, PRV, S. suis, S. aureus, Salmonella, CSFV, PRRSV and PEDV were tested.

The robustness and reproducibility of the qPCR and ddPCR were determined by serially diluted recombinant plasmids. To evaluate intra- and inter-assay reproducibility, each template was tested in triplicate, and the Standard Deviation (SD) and the Coefficient of Variation $(\mathrm{CV})$ were calculated and analyzed statistically.

\subsection{Comparison of ddPCR and qPCR Assay}

The pUC57-APP and pUC57-HPS which serially 10-fold and twofold diluted were used to compare the sensitivity and accuracy between ddPCR and qPCR. The quantitative agreement between ddPCR and qPCR measurements was access by correlation analysis using standard curves of qPCR and ddPCR.

\subsection{Clinical Sample Detection}

One hundred and forty clinical samples and 50 lung tissue samples were used to evaluate the ability of qPCR and ddPCR. The positive detection rate was compared and evaluated the sensitivity of both methods.

\subsection{Statistical Analysis}

The correlations and regressions analysis of the standard curves from qPCR were analyzed by origin 2017 (OriginLab, MA, USA). The copy number of the initial templates from ddPCR was analyzed by QuantaSoft analysis software (Bio-Rad). Kappa statistics were used to evaluate the agreement of clinical detection results between qPCR and ddPCR.

\section{RESULTS}

\subsection{Primer Annealing Temperature Optimizing}

According to the manufacturer's instructions, the optimized annealing temperature of qPCR was $60^{\circ} \mathrm{C}$. For ddPCR assay, the annealing temperature gradients test was carried out at the following temperatures: $60^{\circ} \mathrm{C}, 59.4^{\circ} \mathrm{C}, 58.3^{\circ} \mathrm{C}, 56.3^{\circ} \mathrm{C}, 53.9^{\circ} \mathrm{C}$, $52^{\circ} \mathrm{C}, 50.7^{\circ} \mathrm{C}$, and $50^{\circ} \mathrm{C}$. As shown in Figure 1 , the optimal annealing temperature of $\mathrm{ddPCR}$ was $56.3^{\circ} \mathrm{C}$, which could effectively distinguish the signals between fluorescent channels peaked.

\subsection{Specificity and Reproducibility Test of ddPCR}

The CV is used to reflect the robustness and reproducibility of ddPCR. As shown in Tables 1 and 2, CV values of intra-assay variation was $0.54-6.67 \%$ for A. pleuropneumoniae and $0.64-6.83 \%$ for $H$. parasuis, and inter-assay variation was $0.28-5.33 \%$ for A. pleuropneumoniae and $0.85-4.76 \%$ for $H$. parasuis, which indicated good reproducibility and robustness of ddPCR. The results of specificity test showed the target strains and positive samples were correctly identified and no generating false-positive or falsenegative results were detected. Thus, it confirmed that the primers and probes designed in this study had no non-specific amplification in the genomes on the above pathogens, which indicated that ddPCR had good specificity.

\subsection{Analysis of Standard Curves and Detection Limits}

To assess the accuracy and sensitivity, serial dilutions of recombinant pUC57-APP and pUC57-HPS were tested by both PCR assays (Table 3 and Figure 2). Standard curves and efficacies of qPCR were obtained upon serial 10 -fold dilutions. A. pleuropneumoniae and $H$. parasuis PCR efficacies were 1.064 and 1.143 , respectively. The Limit of Detection (LoD) of duplex qPCR was 105 and 45 copies/ $\mu \mathrm{L}$ for plasmid DNA of A. pleuropneumoniae and $H$. parasuis, respectively. In contrast, the LoD for ddPCR detection of plasmid was 2 copies/ $\mu \mathrm{L}$ for both A. pleuropneumoniae and $H$. parasuis. The LoD for ddPCR detection of $A$. pleuropneumoniae and $H$. parasuis was 50 -fold and 20 -fold better than qPCR, respectively. The correlation coefficient $\left(R^{2}\right)$ was calculated on the mean value of target copy numbers measured in the sensitivity of ddPCR and qPCR, which is used to determine the linearity over the dynamic range. The results were showed that, in the range of quantification, both qPCR and ddPCR have good linearity (Figure 3). 
A

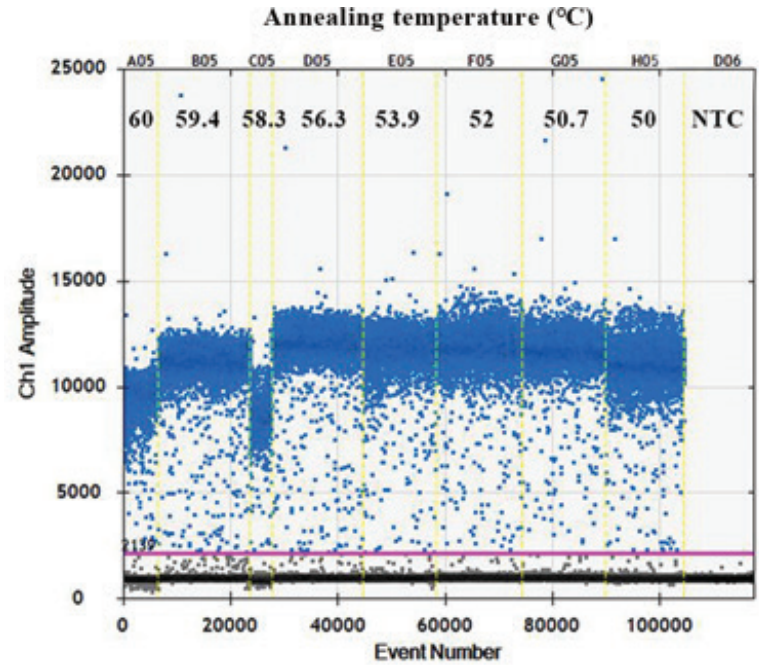

B

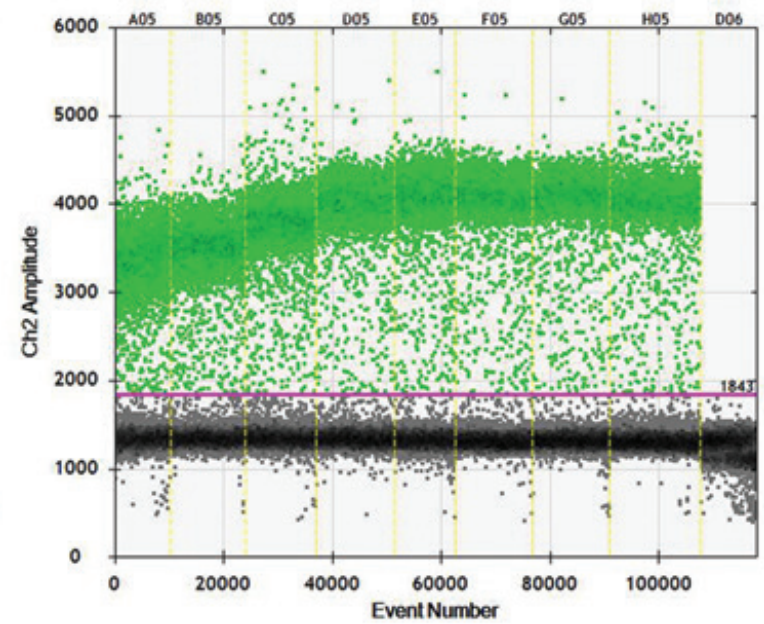

Figure 1 Optimization of annealing temperature for detection of pUC57-APP and pUC57-HPS. (A) Lane A05:60 ${ }^{\circ} \mathrm{C}$; lane $\mathrm{B} 05: 59.4^{\circ} \mathrm{C}$; lane $\mathrm{C} 05: 58.3^{\circ} \mathrm{C}$; lane D05:56. $3^{\circ} \mathrm{C}$; lane E05:53.9 ${ }^{\circ} \mathrm{C}$; lane $\mathrm{F} 05: 52^{\circ} \mathrm{C}$; lane $\mathrm{G} 05: 50.7^{\circ} \mathrm{C}$; lane $\mathrm{H} 05: 50^{\circ} \mathrm{C}$; lane D06: NTC. Blue plots indicate pUC57-APP (FAM signal).

(B) Green plots indicate pUC57-HPS (VIC signal).

Table 1 Robustness and reproducibility of ddPCR assay for A. pleuropneumoniae

\begin{tabular}{|c|c|c|c|c|c|c|}
\hline \multirow{2}{*}{$\begin{array}{l}\text { Concentration of pUC57- } \\
\text { APP (copies/ } \mu \mathrm{L})\end{array}$} & \multicolumn{3}{|c|}{ Intra-assay variation } & \multicolumn{3}{|c|}{ Inter-assay variation } \\
\hline & Mean $($ copies $/ \mu \mathrm{L})$ & SD & $\mathrm{CV}(\%)$ & Mean $($ copies $/ \mu \mathrm{L})$ & SD & $\mathrm{CV}(\%)$ \\
\hline $1.4 \times 10^{5}$ & 7441 & 40.45 & 0.54 & 7457 & 20.79 & 0.28 \\
\hline $1.2 \times 10^{4}$ & 875 & 6.66 & 0.76 & 882 & 12.34 & 1.40 \\
\hline $1.1 \times 10^{3}$ & 102 & 6.56 & 6.43 & 101 & 4.51 & 4.48 \\
\hline 95 & 14 & 0.75 & 5.20 & 16 & 0.55 & 3.52 \\
\hline 46 & 7 & 0.35 & 4.92 & 7 & 0.31 & 4.09 \\
\hline 22 & 2 & 0.15 & 6.67 & 2 & 0.12 & 5.33 \\
\hline
\end{tabular}

Table 2 Robustness and reproducibility of ddPCR assay for $H$. parasuis

\begin{tabular}{|c|c|c|c|c|c|c|}
\hline \multirow{2}{*}{$\begin{array}{l}\text { Concentration of pUC57- } \\
\text { HPS (copies/ } \mu \mathrm{L})\end{array}$} & \multicolumn{3}{|c|}{ Intra-assay variation } & \multicolumn{3}{|c|}{ Inter-assay variation } \\
\hline & Mean (copies/ $\mu \mathrm{L})$ & SD & $\mathrm{CV}(\%)$ & Mean (copies/ $\mu \mathrm{L})$ & SD & CV (\%) \\
\hline $4.8 \times 10^{4}$ & 4863 & 31.00 & 0.64 & 4874 & 43.84 & 0.90 \\
\hline $3.4 \times 10^{3}$ & 746 & 26.69 & 3.58 & 776 & 6.56 & 0.85 \\
\hline $2.4 \times 10^{2}$ & 115 & 5.69 & 4.93 & 137 & 3.06 & 2.22 \\
\hline 17 & 17 & 0.96 & 5.57 & 18 & 0.59 & 3.18 \\
\hline 8 & 8 & 0.51 & 6.47 & 8 & 0.35 & 4.30 \\
\hline 3 & 2 & 0.16 & 6.83 & 2 & 0.10 & 4.76 \\
\hline
\end{tabular}

Table 3 Comparative limit of detection between ddPCR and qPCR assay

\begin{tabular}{|c|c|c|c|c|c|}
\hline \multicolumn{2}{|c|}{ Concentration of plasmids ${ }^{\mathrm{a}}($ copies $/ \mu \mathrm{L})$} & \multicolumn{2}{|c|}{ qPCR (Mean Cq value) } & \multicolumn{2}{|c|}{$\operatorname{ddPCR}^{\mathrm{b}}($ Mean concentration, copies $/ \mu \mathrm{L})$} \\
\hline pUC57-APP & pUC57-HPS & pUC57-APP & pUC57-HPS & pUC57-APP & pUC57-HPS \\
\hline $1.6 \times 10^{6}$ & $6.8 \times 10^{5}$ & 21.4 & 20.5 & NT & NT \\
\hline $1.4 \times 10^{5}$ & $4.8 \times 10^{4}$ & 24.6 & 23.7 & 7441 & 4863 \\
\hline $1.2 \times 10^{4}$ & $3.4 \times 10^{3}$ & 28.0 & 27.4 & 875 & 746 \\
\hline $1.1 \times 10^{3}$ & $2.4 \times 10^{2}$ & 31.3 & 31.1 & 102 & 115 \\
\hline 95 & 17 & 34.0 & 35.1 & 14 & 17 \\
\hline 46 & 8 & ND & ND & 7 & 8 \\
\hline 22 & 3 & ND & ND & 2 & 2 \\
\hline NTC & NTC & ND & ND & ND & ND \\
\hline
\end{tabular}

NTC, no template control; ND, not detected; NT, not tested; Cq, quantification cycle. ${ }^{2}$ Concentration based on 10 -fold and twofold serial dilutions of plasmid. ${ }^{b}$ Concentration based on ddPCR detection. 
A

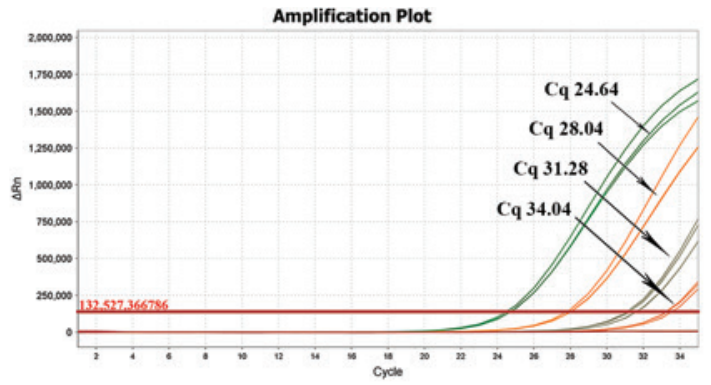

$\mathrm{C}$

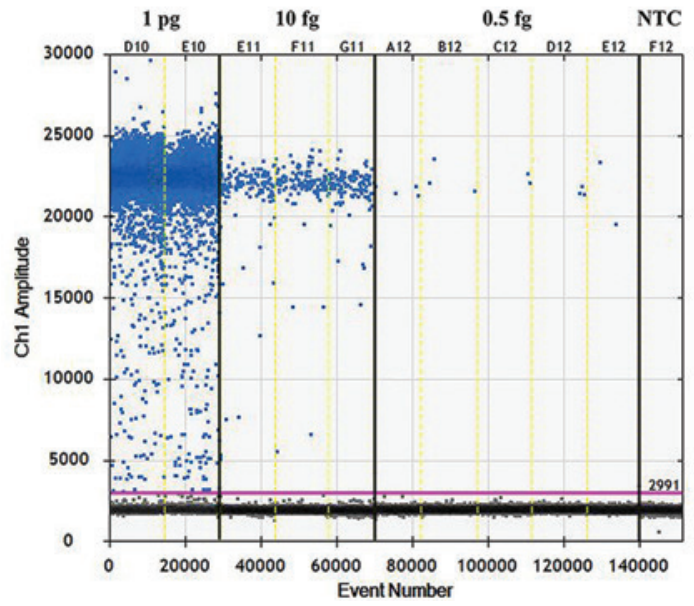

B

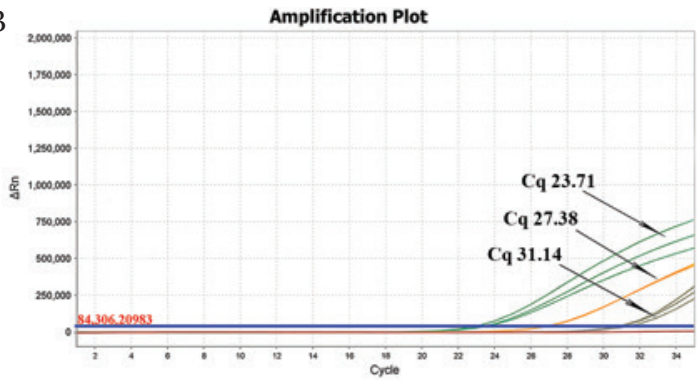

$\mathrm{D}$

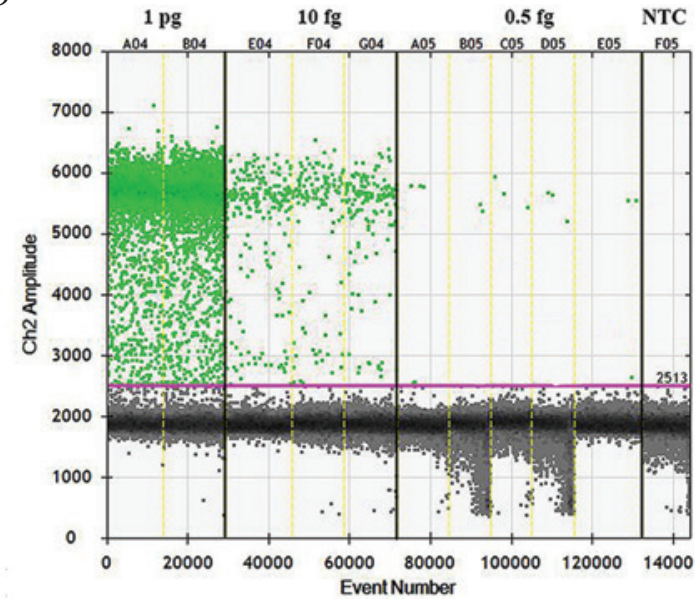

Figure 2 Comparison of the sensitivity of pUC57-APP and pUC57-HPS by RT-PCR and ddPCR. (A) and (B) The real-time fluorescence monitoring of pUC57-APP and pUC57-HPS were performed by qPCR, respectively. (C) and (D) The fluorescence amplitude of pUC57-APP and pUC57-HPS by ddPCR (1D Droplet Plots), respectively. NTC, negative template control.
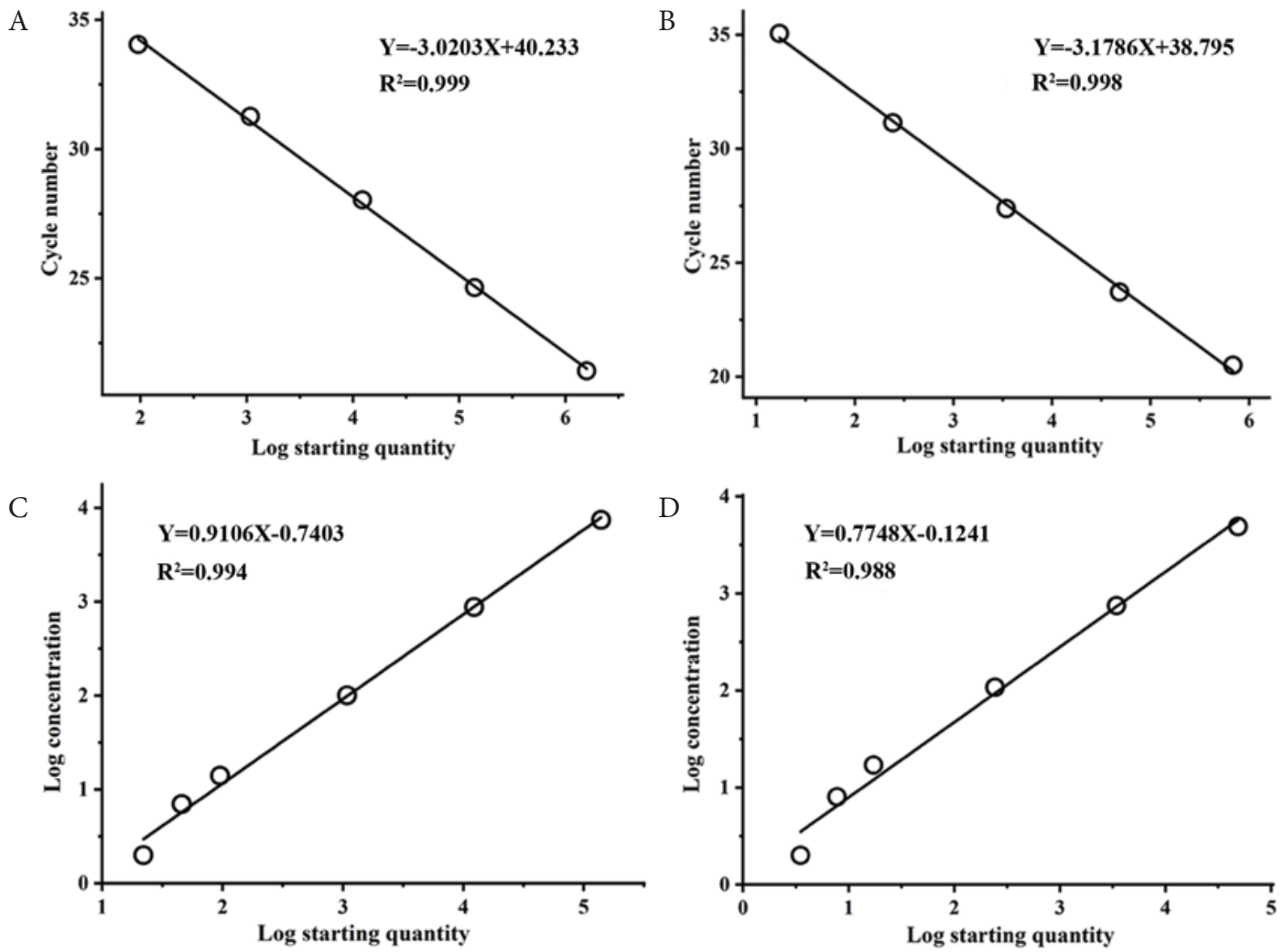

Figure 3 Quantification of serially diluted recombinant pUC57-APP and pUC57-HPS by ddPCR and qPCR. (A) and (B) Standard curves of qPCR were constructed using serial dilution of pUC57-APP and pUC57-HPS, respectively. The quantification correlation of qPCR was obtained by plotting the quantitative cycle value against the log starting concentration. (C) and (D) Standard curves of ddPCR were constructed using serial dilution of pUC57-APP and pUC57-HPS, respectively. The quantification relation of ddPCR between the log determined concentration and the log starting concentration. 


\subsection{Application and Detection of Clinical Samples}

DNA extracted from 50 lung tissue of healthy pigs were confirmed negative by ELISA for A. pleuropneumoniae and $H$. parasuis and used as a negative control. For these negative samples, both qPCR and ddPCR were negative for A. pleuropneumoniae and $H$. parasuis. $12 \mathrm{~A}$. pleuropneumoniae and $16 \mathrm{H}$. parasuis positive clinical samples were confirmed by ELISA and PCR, and both qPCR and dAPCR showed $100 \%$ positive and no false-positive results were shown. Another 112 were suspected A. pleuropneumoniae or $H$. parasuis infected samples. Among 112 clinical suspected samples, the positive detection rate for A. pleuropneumoniae by using qPCR and ddPCR was $7.1 \%$ and $10.7 \%$, respectively; and the positive detection rate for H. parasuis was $8.9 \%$ in $\mathrm{qPCR}$ and $12.5 \%$ in ddPCR, respectively (Tables 4 and 5). All samples accurately detected by qPCR can also be detected by ddPCR, and the suspected clinical samples were further confirmed by ELISA. Compared with qPCR, ddPCR showed a higher positive detection rate, indicating that ddPCR assay is a more efficient method for the diagnose of A. pleuropneumoniae and $H$. parasuis suspected mixed infected clinical samples. The kappa value, which is used to measure agreement between two methods, was 0.781 and 0.814 for A. pleuropneumoniae and $H$. parasuis, indicating that qPCR and ddPCR assays have a good agreement.

\section{DISCUSSION}

The surveillance of A. pleuropneumoniae and $H$. parasuis depends on the rapid and accuracy diagnosis. Conventional laboratory methods are less sensitive, laborious and time-consuming. Because of the precision and specificity of ddPCR, especially in the serial dilutions of genomic DNA from both pathogens, ddPCR is an effective method to detect bacterial DNA [28]. In addition, compared with other PCR methods, the difference of ddPCR is that each template molecule is amplified in an independent reaction chamber generated by water droplets emulsified in oil [26]. Each droplet acts as an independent micro-reactor, so it can achieve highly efficient amplification. Therefore, at the single-molecule resolution level, the sensitivity of ddPCR is higher than that of qPCR.

Table 4 Results of ddPCR and qPCR assay for detection of

A. pleuropneumoniae in clinical suspected samples

\begin{tabular}{lccc}
\hline \multirow{2}{*}{ Detection methods } & \multicolumn{2}{c}{ ddPCR } & Total \\
\cline { 2 - 3 } & Positive & Negative & \\
\hline qPCR & 8 & 0 & 8 \\
& 4 & 100 & 104 \\
& 12 & 100 & 112 \\
\hline
\end{tabular}

Table 5 Results of ddPCR and qPCR assay for detection of $H$. parasuis in clinical suspected samples

\begin{tabular}{lccc}
\hline \multirow{2}{*}{ Detection methods } & \multicolumn{2}{c}{ ddPCR } & Total \\
\cline { 2 - 3 } & Positive & Negative & \\
\hline qPCR & 10 & 0 & 10 \\
& 4 & 98 & 102 \\
& 14 & 98 & 112 \\
\hline
\end{tabular}

In this study, the serially diluted plasmid was used to measure the sensitivity of qPCR and ddPCR. As a novel molecular method, ddPCR has the advantage of absolute quantification detection of nucleic acids without relying on references or calibration curves. Our duplex ddPCR assay was specific and more sensitive than $\mathrm{qPCR}$ in detecting A. pleuropneumoniae and $H$. parasuis simultaneously. The detection of clinical samples that were identified by ELISA confirms that ddPCR has more accurate sensitivity. However, ddPCR is sometimes not as universal as qPCR due to the multiple partitions generated in the test. Furthermore, ddPCR may provide non-linear results when the initial concentration of the sample is too high. As described in the instruction manual of ddPCR, it is not practical for the detection of high concentration with more than $10^{5}$ copies of pathogens in clinical samples. Therefore, qPCR could be used when the pathogen concentration is very high $\left(>10^{5}\right.$ copies in the added template), while ddPCR is acceptable for the clinical samples with a low concentration $(<105$ copies $/ \mu \mathrm{L}$ for A. pleuropneumoniae and 45 copies/ $\mu \mathrm{L}$ for $H$. parasuis in the added template). Nevertheless, the cost of ddPCR reagents is comparatively high compared with qPCR for conventional diagnostic work, which may restrict its use and makes it hard to spread.

In the scope of our knowledge, this is the first assay for sensitive and simultaneous identification of A. pleuropneumoniae and $H$. parasuis by qPCR and ddPCR. In related molecular detection studies, limits of detection of $A$. pleuropneumoniae and $H$. parasuis were reported. A qPCR assay for detecting $H$. parasuis showed a sensitivity of LoD was 9.5-0.83 CFU per reaction for the boiling method of DNA extraction and 47.5-0.42 CFU per reaction for the PrepMan Ultra method; and for detecting A. pleuropneumoniae performed an analytical sensitivity of five colony forming units/reaction [18,29]. A loop-mediated isothermal amplification assay for detecting $H$. parasuis displayed a sensitivity of LoD was $0.2 \mathrm{pg} / \mu \mathrm{L}$ and for detecting A. pleuropneumoniae performed at a sensitivity of $8 \mathrm{CFU}$ per tube [30,31]. A multiplex PCR assay for detecting A. pleuropneumoniae, Pasteurella multocida and $H$. parasuis showed the minimum detection concentration of 100 DNA copies, 10 DNA copies and 7 DNA copies, respectively [32]. In this study, the LoD (2 copies) by duplex ddPCR for both pathogens was lower than the published results. ddPCR may be useful to detect low concentration of clinical tissue samples of A. pleuropneumoniae and $H$. parasuis.

\section{CONCLUSION}

We established a duplex ddPCR assay for A. pleuropneumoniae and $H$. parasuis with higher specificity and greater sensitivity to those of qPCR. The duplex ddPCR assay is accurate for detecting both pathogens in serial dilutions. What's more, the applicability of ddPCR is evaluated in clinical samples, which exhibits a higher positive detection rate than qPCR. The established method in this study can be a new approach to simultaneous quantitative detection of A. pleuropneumoniae and $H$. parasuis, so as to reduce the spread of swine diseases and to certain extent ensure the safety of meat food.

\section{CONFLICTS OF INTEREST}

The authors declare they have no conflicts of interest. 


\section{AUTHORS' CONTRIBUTION}

WW contributed in literature search, experimental data collection and analyses, manuscript writing and editing. LS contributed in conceptualization and project administration. XC contributed in visualization, methodology, supervision, manuscript review.

\section{ACKNOWLEDGMENTS}

This work was supported by National key research and development plan (2016YFD0500600), National Natural Science Foundation of China (31901789), and Fundamental Research Funds for the Central Universities (21618309).

\section{REFERENCES}

[1] Eshitera EE, Githigia SM, Kitala P, Thomas LF, Fèvre EM, Harrison LJS, et al. Prevalence of porcine cysticercosis and associated risk factors in Homa Bay district, Kenya. BMC Vet Res 2012;8:234.

[2] Lai J, Holly Wang H, Ortega DL, Olynk Widmar NJ. Factoring Chinese consumers' risk perceptions into their willingness to pay for pork safety, environmental stewardship, and animal welfare. Food Control 2018;85:423-31.

[3] Han JQ, Trienekens JH, Omta SWF. Relationship and quality management in the Chinese pork supply chain. Int J Prod Econ 2011;134:312-21.

[4] Cohen J, Enserink M. Swine flu. After delays, WHO agrees: the 2009 pandemic has begun. Science 2009;324:1496-7.

[5] Ge S, Li J, Fan X, Liu F, Li L, Wang Q, et al. Molecular characterization of African swine fever virus, China, 2018. Emerg Infect Dis 2018;24:2131-3.

[6] Gottshchalk M, Broes A. Actinobacillus pleuropneumoniae. In: Zimmerman JJ, Karriker LA, Ramirez A, Schwartz KJ, Stevenson GW, Zhang J, editors. Diseases of Swine. 11th ed. Hoboken, NJ: John Wiley \& Sons, Inc; 2019, pp. 749-66.

[7] Oliveira S, Pijoan C. Haemophilus parasuis: new trends on diagnosis, epidemiology and control. Vet Microbiol 2004;99:1-12.

[8] González W, Giménez-Lirola LG, Holmes A, Lizano S, Goodell C, Poonsuk K, et al. Detection of Actinobacillus pleuropneumoniae ApxIV toxin antibody in serum and oral fluid specimens from pigs inoculated under experimental conditions. J Vet Res 2017;61:163-71.

[9] Deslandes V, Denicourt M, Girard C, Harel J, Nash JH, Jacques M. Transcriptional profiling of Actinobacillus pleuropneumoniae during the acute phase of a natural infection in pigs. BMC Genom 2010;11:98.

[10] Guo Ll, Zhang Jm, Xu Cg, Ren T, Zhang B, Chen Jd, et al. Detection and characterization of $\beta$-Lactam resistance in Haeophilus parasuis strains from pigs in south China. J Integr Agric 2012;11:116-21.

[11] Fu S, Ou J, Zhang M, Xu J, Liu H, Liu J, et al. The live attenuated Actinobacillus pleuropneumoniae triple-deletion mutant $\triangle a p x I C \triangle a p x I I C \triangle a p x I V-O R F 1$ strain, SLW05, Immunizes pigs against lethal challenge with Haemophilus parasuis. Clin Vaccine Immunol 2013;20:134-9.

[12] Jung M, Won H, Shin MK, Oh MW, Shim S, Yoon I, et al. Development of Actinobacillus pleuropneumoniae ApxI, ApxII, and ApxIII-specific ELISA methods for evaluation of vaccine efficiency. J Vet Sci 2019;20:e2.
[13] Álvarez-Estrada Á, Martínez-Martínez S, Martín CBG, GarcíaIglesias MJ, Pérez-Martínez C, Yubero-Delgado S, et al. Immunogenic characterization of vaccines based on Haemophilus parasuis Nagasaki strain, OmpP2, OmpP5 and OmpD15, in colostrum-deprived pigs experimentally challenged with the same strain. Res Vet Sci 2018;119:292-301.

[14] Yang L, Liu L, Cao SJ, Wen XT, Huang XB. Development and application of a semi-nested PCR assay for detection of porcine Actinobacillus pleuropneumoniae. Chin Vet Sci 2009;39:790-5.

[15] Zehr ES, Tabatabai LB. Detection of a bacteriophage gene encoding a Mu-like portal protein in Haemophilus parasuis reference strains and field isolates by nested polymerase chain reaction. J Vet Diagn Invest 2011;23:538-42.

[16] Marois-Créhan C, Lacouture S, Jacques M, Fittipaldi N, Kobisch M, Gottschalk M. Development of two real-time polymerase chain reaction assays to detect Actinobacillus pleuropneumoniae serovars 1-9-11 and serovar 2. J Vet Diagn Invest 2014;26:146-9.

[17] Mcdowall R, Slavic D, MacInnes JI, Cai HY. Evaluation of a realtime polymerase chain reaction assay of the outer membrane protein P2 gene for the detection of Haemophilus parasuis in clinical samples. Can J Vet Res 2014;78:150-2.

[18] Li R, Wang J, Liu L, Zhang R, Hao X, Han Q, et al. Direct detection of Actinobacillus pleuropneumoniae in swine lungs and tonsils by real-time recombinase polymerase amplification assay. Mol Cell Probe 2019;45:14-18.

[19] Kawasaki S, Fratamico PM, Kamisaki-Horikoshi N, Okada Y, Takeshita K, Sameshima T, et al. Development of the multiplex PCR detection kit for Salmonella spp., Listeria monocytogenes, and Escherichia coli O157:H7. Jpn Agric Res Q 2011;45:77-81.

[20] He P, Chen Z, Luo J, Wang H, Yan Y, Chen LX, et al. Multiplex real-time PCR assay for detection of pathogenic Vibrio parahaemolyticus strains. Mol Cell Probe 2014;28:246-50.

[21] Cao S, Chen H, Zhao J, Lü J, Xiao S, Jin M, et al. Detection of porcine circovirus type 2, porcine parvovirus and porcine pseudorabies virus from pigs with postweaning multisystemic wasting syndrome by multiplex PCR. Vet Res Commun 2005;29:263-9.

[22] Chandra M, Cheng P, Rondeau G, Porwollik S, McClelland M. A single step multiplex PCR for identification of six diarrheagenic E. coli pathotypes and Salmonella. Int J Med Microbiol 2013;303:210-16.

[23] Wang Y, Feng Y, Zheng WL, Noll L, Porter E, Potter M, et al. A multiplex real-time PCR assay for the detection and differentiation of the newly emerged porcine circovirus type 3 and continuously evolving type 2 strains in the United States. J Virol Methods 2019;269:7-12.

[24] Alanio A, Sturny-Leclère A, Benabou M, Guigue N, Bretagne S. Variation in copy number of the 28S rDNA of Aspergillus fumigatus measured by droplet digital PCR and analog quantitative real-time PCR. J Microbiol Methods 2016;127:160-3.

[25] Kelley K, Cosman A, Belgrader P, Chapman B, Sullivan DC. Detection of Methicillin-Resistant Staphylococcus aureus by a duplex droplet digital PCR assay. J Clin Microbiol 2013;51:2033-9.

[26] Bian X, Jing F, Li G, Fan X, Jia C, Zhou H, et al. A microfluidic droplet digital PCR for simultaneous detection of pathogenic Escherichia coli O157 and Listeria monocytogenes. Biosens Bioelectron 2015;74:770-7.

[27] Ren M, Lin H, Chen S, Yang M, An W, Wang Y, et al. Detection of pseudorabies virus by duplex droplet digital PCR assay. J Vet Diagn Invest 2018;30:105-12. 
[28] He L, Simpson DJ, Gänzle MG. Detection of enterohaemorrhagic Escherichia coli in food by droplet digital PCR to detect simultaneous virulence factors in a single genome. Food Microbiol 2020;90:103466.

[29] Turni C, Pyke M, Blackall PJ. Validation of a real-time PCR for Haemophilus parasuis. J Appl Microbiol 2010;108:1323-31.

[30] Wei XY, Xu RT, Wang CG, Zhang T. Loop-mediated isothermal amplification targeting OMP P2 gene for detection of Haemophilus parasuis. Int J Agric Biol 2018;20:230-4.
[31] Wang Y, Chen P, Guo HB, Chen Y, Liu H, He QH. Loop-mediated isothermal amplification targeting the apxIVA gene for detection of Actinobacillus pleuropneumoniae. FEMS Microbiol Lett 2009;300:83-9.

[32] Hričínová M, Holoda $E$, Mudroňová $D$, Ondrašovičová S. Multiplex PCR assay for detection of Actinobacillus pleuropneumoniae, Pasteurella multocida and Haemophilus parasuis in lungs of pigs from a slaughterhouse. Folia Microbiol 2010;55: 635-40. 